\title{
Plenary Speaker Lockwood to Address Materials Aspect of Volcanoes at MRS Spring Meeting
}

"Volcanoes have been my professional focus and passion for over 20 years," said volcanologist John P. "Jack" Lockwood, President and Chair of the Board of Geohazards Consultants International, Inc. (GCI). Lockwood will present his plenary talk, "Volcanoes: The Ultimate Materials Source," on Monday, April 13 at 6:00 p.m. in Salon 7, San Francisco Marriott, at the 1998 MRS Spring Meeting in San Francisco.

In more than 15 years of volcanic-crisis and disaster assignments with the U.S. Geological Survey (USGS) and the USAID Office of Foreign Disaster Assistance (OFDA), Lockwood has studied dozens of volcanos, from Gamalama and Nyragongo to Mt. Pinatubo. Among his clients are the U.S. Air Force, the U.S. Army Corps of Engineers, the U.S. National Oceanic and Atmospheric

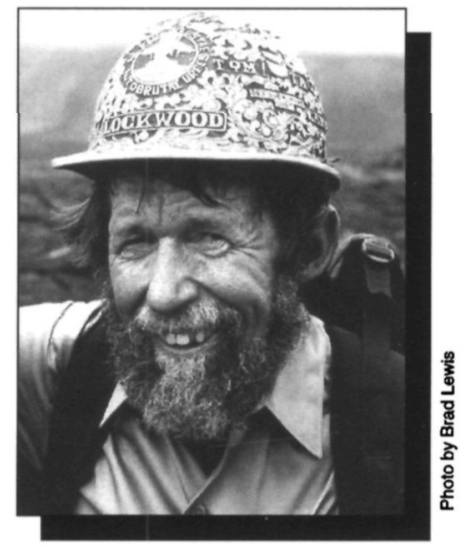

Administration (NOAA), and the U.S. Agency for International Development.

In addition to overseeing the operations of GCI, a field-oriented company based in Volcano, Hawaii, specializing in geohazards appraisals and risk reduction studies, especially in remote or less-developed areas, Lockwood serves as a technical advisor to media projects in film, video, and print. He was the lead scientific consultant for the recently released Universal Pictures film Dante's Peak.

Lockwood holds a bachelor's degree in geology from the University of CaliforniaRiverside, and earned his $\mathrm{PhD}$ degree in geology from Princeton University in 1966. Since 1980 he has been an Adjunct Professor at the University of Hawaii, teaching volcanology classes. He is a fellow of the Geological Society of America, and a member of the International Association of Volcanology and Chemistry of the Earth's Interior, the American Geophysical Union, and Sigma Xi. Lockwood's website is http://www.volcanologist.com. [MाR

\section{MRS Spring Meeting • April 13 - $17 \bullet$ San Francisco, CA}

\section{Sympusium Tutorials}

\begin{tabular}{|c|c|c|}
\hline Sunday • April 12 & Monday • April 13 & Wednesday • April 15 \\
\hline $\begin{array}{c}\text { Symposium G } \\
\text { 1:00 p.m. - 5:00 p.m. } \\
\text { STG: Organic Light-Emitting Diodes } \\
\text { Room: Pacific H }\end{array}$ & $\begin{array}{c}\text { Symposium A } \\
\text { 8:30 a.m. - 4:30 p.m. } \\
\text { STA: Amorphus Silicon Materials } \\
\text { and Devices for Large-Area } \\
\text { Electronics } \\
\text { Room: Salon 11/12 }\end{array}$ & $\begin{array}{c}\text { Symposium D } \\
\text { 1:30 p.m. - 5:00 p.m. } \\
\text { STD: Defects/mpurities and } \\
\text { Gettering in Sillicon Science } \\
\text { and Technology } \\
\text { Room: Golden Gate B2 }\end{array}$ \\
\hline $\begin{array}{c}\text { Symposium L. } \\
\text { 1:30 p.m. - 4:30 p.m. } \\
\text { STL: Physics and Materials for } \\
\text { Ultrahigh-Density Recording } \\
\text { Room: Pacific I }\end{array}$ & $\begin{array}{c}\text { Symposia B/C } \\
\text { 1:30 p.m. - 5:00 p.m. } \\
\text { STB/C: Flat Panel Display Materials } \\
\text { and Large-Area Processing } \\
\text { Room: Nob Hill B/C }\end{array}$ & \\
\hline $\begin{array}{c}\text { Symposium T } \\
\text { 1:00 p.m. - 5:00 p.m. } \\
\text { STT: Measuring Mechanical } \\
\text { Propertles in the Nanometer } \\
\text { Regime } \\
\text { Room: Pacific J }\end{array}$ & $\begin{array}{c}\text { Symposium S } \\
\text { 8:00 a.m. - 4:00 p.m. } \\
\text { STS: Basic Principles and } \\
\text { Applications of Scanning Probe } \\
\text { Microscopy } \\
\text { Room: Salon 5/6 }\end{array}$ & \\
\hline
\end{tabular}


Place your order today for proceedings of the 1990 MRS Spring Meeting in San Francisco and SAVE!

APRIL 15-17

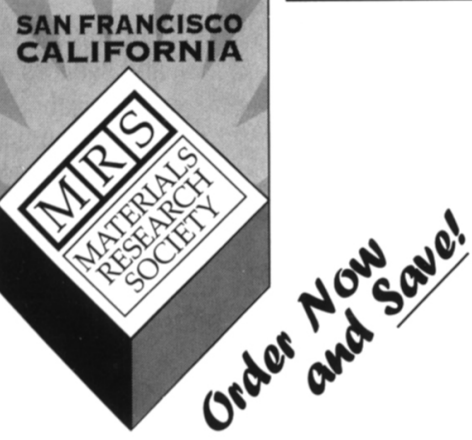

A: Amorphous and Microcrystalline Silicon Technology-1998

Editors: S. Wagner, M. Hack, H.M. Branz, R. Schropp, I. Shimizu

$\begin{array}{ccc}\text { ISBN: } 1-55899-413-0 & & \text { Code: } 507-B \\ \$ 60.00 & \$ 72.00 & \text { MRS Member } \\ \$ 69.00 & \$ 83.00 & \text { U.S. List } \\ \$ 79.00 & \$ 95.00 & \text { Non-U.S. }\end{array}$

B: Flat-Panel Display Materials-1998

Editors: G. Parsons, T.S. Fahlen, S. Morozumi, C. Seager, C-C. Tsai

ISBN: 1-55899-414-9

Code: $508-\mathrm{B}$

$\begin{array}{lll}\$ 57.00 & \$ 69.00 & \text { MRS Member } \\ \$ 66.00 & \$ 79.00 & \text { U.S. List } \\ \$ 75.00 & \$ 91.00 & \text { Non-U.S. }\end{array}$

C: Materials Issues in Vacuum Microelectronics Editors: W. Zhu, L.S. Pan, T.E. Felter, C. Holland ISBN: 1-55899-415-7 Code: 509-B $\begin{array}{lll}\$ 53.00 & \$ 64.00 & \text { MRS Member } \\ \$ 61.00 & \$ 74.00 & \text { U.S. List } \\ \$ 70.00 & \$ 85.00 & \text { Non-U.S. }\end{array}$

D: Defect and Impurity Engineered Semiconductors and Devices II

Editors: S. Ashok, J. Chevallier, K. Sumino, B.L. Sopori, W. Goetz

$\begin{array}{ccc}\text { ISBN: } 1-55899-416-5 & \text { Code: } 510-B \\ \$ 59.00 & \$ 71.00 & \text { MRS Member } \\ \$ 68.00 & \$ 81.00 & \text { U.S. List } \\ \$ 78.00 & \$ 94.00 & \text { Non-U.S. }\end{array}$

E: Low-Dielectric Constant Materials IV Editors: C. Chiang, J.T. Wetzel, T-M. Lu, P.S. Ho ISBN: 1-55899-417-3 Code: 511-B
$\$ 52.00$
$\$ 60.00$
$\$ 63.00$
MRS Member
$\$ 69.00$
$\$ 33.00$
Non-U.S.

F: Wide-Bandgap Semiconductors for High Power, High Frequency and High Temperature Editors: S. Denbaars, M.S. Shur, J. Palmour, M. Spencer ISBN: 1-55899-418-1
$\$ 59.00$
$\$ 68.00$
$\$ 71.00$
MRS Member
$\$ 78.00$
$\$ 94.00$

Code: $512-\mathrm{B}$

H: Hydrogen in Semiconductors and Metals Editors: N.H. Nickel, W.B. Jackson, R.C. Bowman

ISBN: 1-55899-419-X Code: 513-B

$\begin{array}{lll}\$ 57.00 & \$ 69.00 & \text { MRS Member } \\ \$ 66.00 & \$ 79.00 & \text { U.S. List } \\ \$ 75.00 & \$ 91.00 & \text { Non-U.S. }\end{array}$

I: Advanced Interconnects and Contact Materials and Processes for Future Integrated Circuits

Editors: S.P. Murarka, D.B. Fraser, M. Eizenberg, R. Tung, R. Madar

$\begin{array}{ccc}\text { ISBN: } 1-55899-420-3 & & \text { Code: } 514-B \\ \$ 55.00 & \$ 66.00 & \text { MRS Member } \\ \$ 63.00 & \$ 76.00 & \text { U.S. List } \\ \$ 73.00 & \$ 88.00 & \text { Non-U.S. }\end{array}$

$\mathrm{J}$ : Electronic Packaging Materials Science $\mathrm{X}$ Editors: D.J. Belton, R. Pearson, M. Gaynes, E.G. Jacobs ISBN: 1-55899-421-1

$\begin{array}{lll}\$ 5:-55899-421-1 & \text { Code: } 515-B \\ \$ 53.00 & \$ 64.00 & \text { MRS Member } \\ \$ 61.00 & \$ 74.00 & \text { U.S. List } \\ \$ 70.00 & \$ 85.00 & \text { Non-U.S. }\end{array}$

K: Materials Reliability in Microelectronics VIII Editors: T. Marieb, J. Bravman, M.A. Korhonen, J.R. Lloyd ISBN: 1-55899-422-X

$\begin{array}{lll}\$ 57.00 & \$ 69.00 & \text { MRS Member } \\ \$ 66.00 & \$ 79.00 & \text { U.S. List } \\ \$ 75.00 & \$ 91.00 & \text { Non-U.S. }\end{array}$

L/M:High-Density Magnetic Recording and Integrated Magneto-0ptics: Materials and Devices

Editors: K. Rubin, J.A. Bain, T. Nolan, D. Bogy, B.J.H. Stadler, M. Levy, J.P. Lorenzo, M. Mansuripur, Y. Okamura, R. Wolfe ISBN: 1-55899-423-8

$\begin{array}{lll}\$ 59.00 & \$ 71.00 & \text { MRS Membe } \\ \$ 68.00 & \$ 81.00 & \text { U.S. List } \\ \$ 78.00 & \$ 94.00 & \text { Non-U.S. }\end{array}$

N: Microelectromechanical Structures for Materials Research

Editors: S.B. Brown, C. Muhlstein, P. Krulevitch,

G.C. Johnston, R.T. Howe, J.R. Gilbert

ISBN: 1-55899-424-6

$\begin{array}{lll}: 1-55899-424-6 & \text { Code: } 518-B \\ \$ 57.00 & \$ 69.00 & \text { MRS Member } \\ \$ 66.00 & \$ 79.00 & \text { U.S. List } \\ \$ 75.00 & \$ 91.00 & \text { Non-U.S. }\end{array}$

0: Organic/Inorganic Hybrid Materials

Editors: R.M. Laine, C. Sanchez, E. Giannelis, C.J. Brinker

ISBN: 1-55899-425-4 Code: 519-B

$\begin{array}{lll}\$ 59.00 & \$ 71.00 & \text { MRS Member } \\ \$ 68.00 & \$ 81.00 & \text { U.S. List } \\ \$ 78.00 & \$ 94.00 & \text { Non-U.S. }\end{array}$

P: Nanostructured Powders and Their Industrial Application

Editors: G. Beaucage, J.E. Mark, G. Burns, H. Duen-Wu

ISBN: 1-55899-426-2

$\begin{array}{lll}\$ 55.00 & \$ 66.00 & \text { MRS Member } \\ \$ 63.00 & \$ 76.00 & \text { U.S. List } \\ \$ 73.00 & \$ 88.00 & \text { Non-U.S. }\end{array}$

R: Porous and Cellular Materials for Structural Applications

Editors: D.S. Schwartz, D.S. Shih, H.N.G. Wadley, A.G. Evans

ISBN: 1-55899-427-0

$\begin{array}{lll}\$ 1-55899-427-0 & & \text { Code: } 521-B \\ \$ 57.00 & \$ 69.00 & \text { MRS Member } \\ \$ 66.00 & \$ 79.00 & \text { U.S. List } \\ \$ 75.00 & \$ 91.00 & \text { Non-U.S. }\end{array}$

$\mathrm{T}$ : Fundamentals of Nanoindentation and Nanotribology

Editors: N.R. Moody, W.W. Gerberich, S.P. Baker, N. Burnham ISBN: $1-55899-428-9$

$\begin{array}{lll}\$ 57.00 & \$ 69.00 & \text { Code: } 522-B \\ \$ 66.00 & \$ 79.00 & \text { MRS Member } \\ \$ 75.00 & \$ 91.00 & \text { U.S. List } \\ & & \text { Non-U.S. }\end{array}$

U: Electron Microscopy of Semiconducting Materials and ULSI Devices

Editors: C. Hayzelden, F.M. Ross, C.J.D. Hetherington

ISBN: 1-55899-429-7

Code: $523-\mathrm{B}$

$\begin{array}{lll}\$ 53.00 & \$ 64.00 & \text { MRS Member } \\ \$ 61.00 & \$ 74.00 & \text { U.S. List } \\ \$ 70.00 & \$ 85.00 & \text { Non-U.S. }\end{array}$

V: Application of Synchrotron Radiation Techniques to Materials Science IV

Editors: S.M. Mini, D.L. Perry, S.R. Stock, L.J. Terminello ISBN: 1-55899-430-0 Code: 524-B

$\begin{array}{lll}\$ 53.00 & \$ 64.00 & \text { MRS Member } \\ \$ 61.00 & \$ 74.00 & \text { U.S. List } \\ \$ 70.00 & \$ 85.00 & \text { Non-U.S. }\end{array}$

W: Rapid Thermal and Integrated Processing VII Editors: M.C. Öztürk, F. Roozeboom, P.J. Timans, S.H. Pas ISBN: 1-55899-431-9

$\begin{array}{lll}\$ 53.00 & \$ 64.00 & \text { MRS Member } \\ \$ 61.00 & \$ 74.00 & \text { U.S. List } \\ \$ 70.00 & \$ 85.00 & \text { Non-U.S. }\end{array}$

Y: Advances in Laser Ablation of Materials Editors: R.K. Singh, D.H. Lowndes, D.B. Chrisey,

J. Narayan, T. Kawai, E. Fogarassy

ISBN: 1-55899-432-7

$\begin{array}{lll}\$ 55.00 & \$ 66.00 & \text { MRS Membe } \\ \$ 63.00 & \$ 76.00 & \text { U.S. List } \\ \$ 73.00 & \$ 88.00 & \text { Non-U.S. }\end{array}$

Z: Diffusion Mechanisms in Crystalline Materials Editors: Y. Mishin, N.E.B. Cowern, C.R.A. Catlow, D. Farkas, G. Vogl

ISBN: 1-55899-433-5

$\begin{array}{lll}\$ 60.00 & \$ 72.00 & \text { MRS Membe } \\ \$ 69.00 & \$ 83.00 & \text { U.S. List } \\ \$ 79.00 & \$ 95.00 & \text { Non-U.S. }\end{array}$

AA: Mechanisms and Principles of Epitaxial Growth in Metallic Systems

Editors: L.T. Wille, C.P. Burmester, K. Terakura, G. Comsa, E.D. Williams

ISBN: $1-55899-434-3 \quad$ Code: $528-B$

$\begin{array}{lll}\$ 53.00 & \$ 64.00 & \text { MRS Member } \\ \$ 61.00 & \$ 74.00 & \text { U.S. List } \\ \$ 70.00 & \$ 85.00 & \text { Non-U.S. }\end{array}$

BB: Computational and Mathematical Models of Microstructural Evolution

Editors: J.W. Bullard, R. Kalia, M. Stoneham, L-Q. Chen

ISBN: 1-55899-435-1

$\begin{array}{lll}\$ 55.00 & \$ 66.00 & \text { MRS Memb } \\ \$ 63.00 & \$ 76.00 & \text { U.S. List } \\ \$ 73.00 & \$ 88.00 & \text { Non-U.S. }\end{array}$

CC: Biomaterials Regulating Cell Function and Tissue Development

Editors: D. Mooney, A.G. Mikos, K.E. Healy, Y. Ikada,

R.C. Thomson

ISBN: 1-55899-436-X

$\begin{array}{ll}\$ 57.00 & \$ 69.00 \\ \$ 66.00 & \$ 79.00 \\ \$ 75.00 & \$ 91.00\end{array}$

Code: $530-\mathrm{B}$

MRS Member

U.S. List

Non-U.S.

DD: Reliability of Photonics Materials and Structures

Editors: E. Suhir, M. Fukuda, C.R. Kurkjian

ISBN: 1-55899-437-8

$\begin{array}{lll}\$ 57.00 & \$ 69.00 & \text { MRS Memb } \\ \$ 66.00 & \$ 79.00 & \text { U.S. List } \\ \$ 75.00 & \$ 91.00 & \text { Non-U.S. }\end{array}$

EE: Silicon Front-End TechnologyMaterials Processing and Modelling

Editors: N.E.B. Cowern, D. Jacobson, P. Griffin,

P. Packan, R. Webb

ISBN: $1-55899-438-6$

$\begin{array}{lll} & & \text { Code: } 532-\mathrm{B} \\ \$ 57.00 & \$ 69.00 & \text { MRS Membe } \\ \$ 66.00 & \$ 79.00 & \text { U.S. List } \\ \$ 75.00 & \$ 91.00 & \text { Non-U.S. }\end{array}$

FF: Epitaxy and Applications of Si-Based Heterostructures

Editors: E.A. Fitzgerald, P.M. Mooney, D.C. Houghton

ISBN: 1-55899-439-4 Code: 533-B

$\begin{array}{lll}\$ 58.00 & \$ 70.00 & \text { MRS Member } \\ \$ 67.00 & \$ 80.00 & \text { U.S. List } \\ \$ 77.00 & \$ 92.00 & \text { Non-U.S. }\end{array}$

These books are scheduled for publication by fall or early winter 1998.

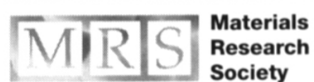

506 Keystone Drive, Warrendale, PA 15086-7573 U.S.A. Phone: 724-779-3003 • Fax: 724-779-8313

E-mail: info@mrs.org • http://www.mrs.org/books/ 


\section{SPRING MEETING}

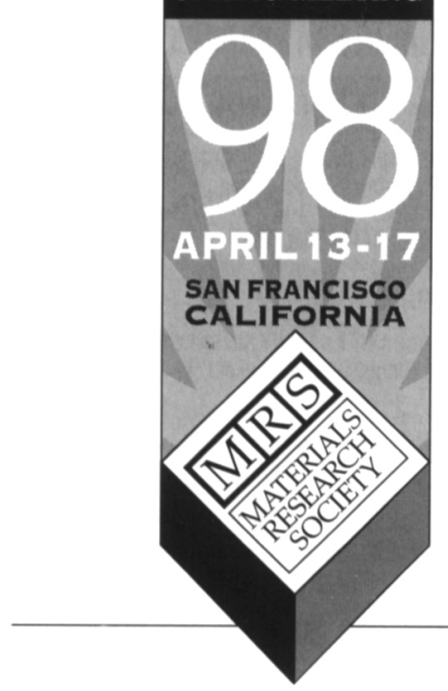

Partial List of 1998 Spring Exhibitors:

A \& N Corporation

ABB Extrel

Academic Press

Accurel-MAG (Materials Analysis Group)

Accurel Systems International Corporation

Aetrium Incorporated

AIXTRON, Inc.

AJA International, Inc.

Aldrich Chemical Company, Inc.

American Institute of Physics, Inc.

APD Cryogenics-IGC

ASTeX/Applied Science and Technology, Inc.

Atom Sciences

Australian Scientific Instruments

Bede Scientific Incorporated

Blake Industries, Inc.

BOC Edwards Vacuum Technology

Burleigh Instruments, Inc.

CERAC, Inc

$\mathrm{CHA}$ Industries

Chemat Technology, Inc.

Commonwealth Scientific Corporation

Creekside Technologies

Cryomech, Inc.

DCA Instruments, Inc.

Denton Vacuum, Inc.

Digital Instruments, Inc.

Duniway Stockroom Corp.

Eagle-Picher Industries, Inc.

EDAX Inc.

Edge Scientific Instrument Company, LLC

Elsevier Science

EMCORE Corporation

Epion Corporation

ERG Materials and Aerospace

ESM Software

Charles Evans \& Associates

FEI Components Group

E.A. Fischione Instruments, Inc.

Fujikin of America, Inc.

Gatan, Inc.

Gelest Inc.

General Vacuum, Inc.

Heraeus Amersil Inc.

\section{San Francisen Marriont Hotel}

\section{Salon 8 and Salon 9 \\ April 14-16, 1998}

The MRS Exhibit, held in conjunction with the 1998 MRS Spring Meeting, will encompass the full spectrum of equipment, instrumentation, products, software, publications and services for materials research. As always, the exhibit will closely parallel the nature of the technical symposia, and the program has been arranged to allow meeting participants ample opportunity to visit the exhibit. MRS encourages attendees to visit the exhibit by scheduling coffee breaks, deli-style lunches, and a meeting-wide reception in the exhibit hall.

\section{Exhibit Hours:}

Tuesday, April 14

$11: 30 \mathrm{am}-6: 30 \mathrm{pm}$

Wednesday, April 15

$9: 30 \mathrm{am}-$

6:00 pm

Thursday, April 16.

9:30 am - 1:30 pm

Complimentary reception will be held in the exhibit hall on Tuesday evening from 5:00 pm to 6:30 pm.
High Voltage Engineering Europa B.V.

Hitachi Scientific Instruments

Huntington Mechanical Laboratories, Inc.

Hysitron Incorporated

IBM Analytical Services

InnoVac Corporation

Instruments S.A., Inc.

Insulator Seal Incorporated

Ion Tech, Inc.

Janis Research Company, Inc.

JEOL USA, Inc.

Keithley Instruments, Inc.

Kevex X-Ray, Inc.

KLA-Tencor

Kluwer Academic Publishers

Kratos Analytical Inc.

Ladd Research Industries, Inc.

Lake Shore Cryotronics, Inc.

Lambda Physik, Inc.

Lasertec U.S.A., Inc.

Kurt J. Lesker Company

Logitech Product Group

Luxel Corporation

LUXTRON Corporation

Magnet Sales \& Manufacturing

MARCH Instruments, Inc.

M. Braun, Inc.

MDC Vacuum Products Corporation

Micrion Corporation

Micro Photonics, Inc.

MMR Technologies, Inc.

Molecular Simulations, Inc.

MTI Corporation

MVSystems, Inc.

Nano Instruments, Inc.

National Electrostatics Corporation

National High Magnetic Field Laboratory

Neocera, Inc.

NFT-Nanofilm Technologie GmbH

Nicolet Instrument Corporation

NORAN Instruments, Inc.

Nor-Cal Products, Inc.

NYS Center for Advanced

Thin Film Technology

Omicron Associates
Osmic, Inc.

Oxford Applied Research

Oxford Instruments America, Inc. Microanalysis Group

Oxford Instruments America, Inc.

Research Instruments

Park Scientific Instruments

Ted Pella, Inc.

Philips Analytical

Philips Electron Optics Inc.

Photonics Spectra

Phytron Instruments, Inc.

Princeton Instruments, Inc.

Princeton Scientific Corp.

Pure Tech Incorporated

QualiTau

Quesant Instrument Corporation

Renishaw Inc.

Research and PVD Materials Corporation

RJ Lee Instruments, Ltd.

RMC

SKION Corporation

South Bay Technology, Inc.

SPI Supplies/Structure Probe, Inc.

Springer-Verlag New York, Inc.

Staib Instruments

Strem Chemicals, Inc.

SURFACE

Surface/Interface, Inc.

SurForce Corporation

SVT Associates, Inc.

TECHNOTRADE International, Inc.

TexSEM Laboratories, Inc. (TSL)

Thermionics Vacuum Products

TherMold Partners L.P.

TopoMetrix Corp.

Union Carbide Crystal Products

Vacuum Atmospheres Company

Varian Vacuum Products

VAT, Inc.

Virginia Semiconductor, Inc.

Voltaix, Inc.

$\mathrm{X}$-ray Instrumentation Associates 


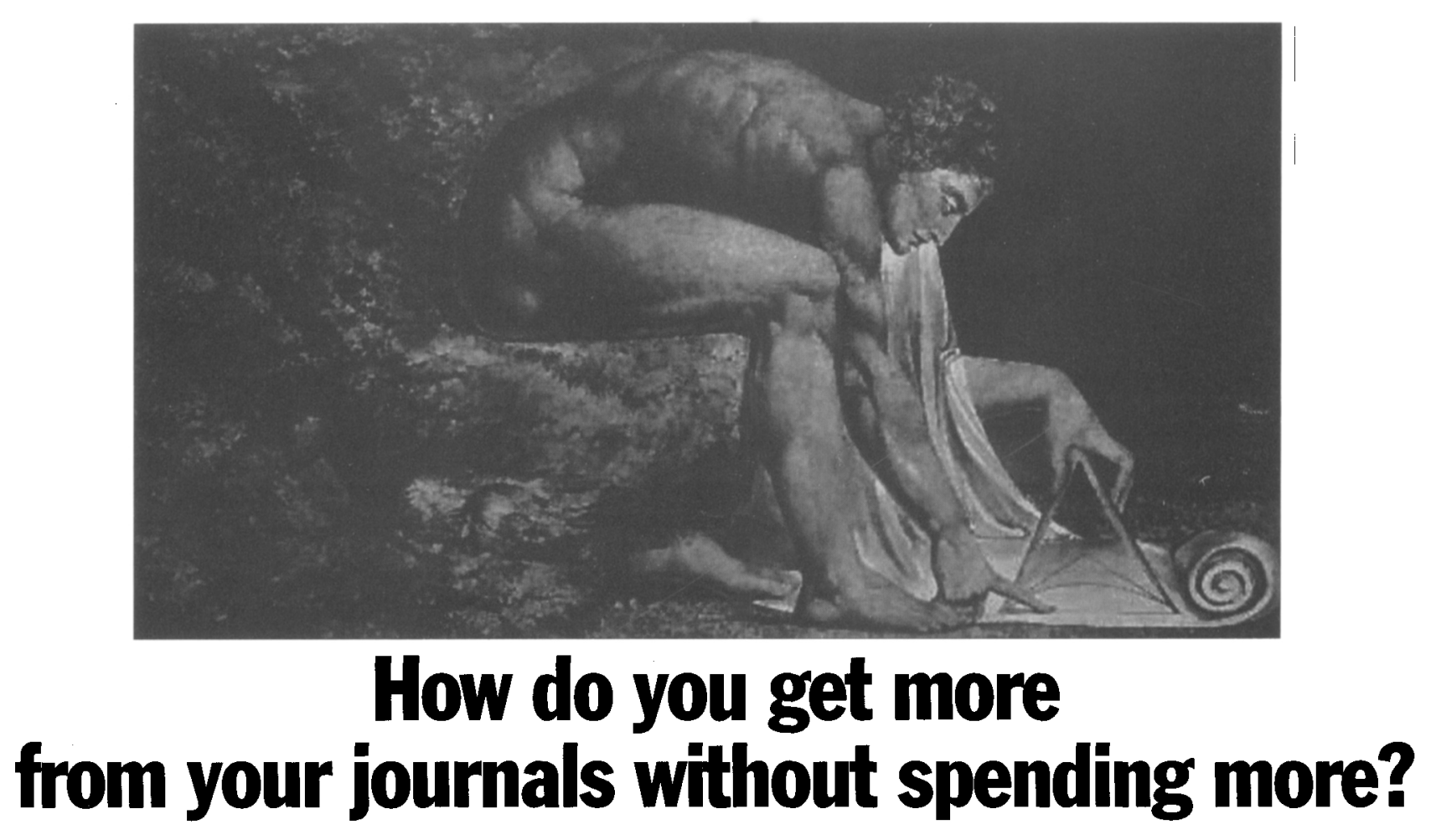

Physicists are used to dealing with difficult questions. They even seem to enjoy the kind of problem which their colleagues from other disciplines might be tempted to dismiss as a hopeless contradiction in terms. Perhaps that's why staff at Institute of Physics Publishing (IoP)have addressed themselves with such relish to the question of how to add even more value to their journals.

\section{Electronic Joumals with HyperCite ${ }^{\mathrm{TM}}$ technology}

How has Institute of Physics Publishing addressed this problem? In 1996 they became one of the first publishers to release their entire journals list via the World Wide Web.

In 1997 they launched their upgraded Electronic Journals service with HyperCite ${ }^{\mathrm{TM}}$. HyperCite ${ }^{\mathrm{TM}}$ enables IoP Electronic Journal users to find papers cited by, and papers citing their selected articles through links from references into a range of full text and abstract resources.

\section{New five year full text archive}

The 1998 solution looks even more impressive. Now all Institute of Physics Publishing Electronic Journal users at - registered sites, whether new or existing customers, will benefit from online access to a five year full text archive (1993 - 1997). Add this to the groundbreaking research for the current year, and you get online access to over five years of full text for subscribed journal titles.
The resulting Electronic Journals service, with the combined features of HyperCite ${ }^{\mathrm{TM}}$ and IoP's extended full text archive, will provide researchers with an additional and expanding five year citation window onto the community's literature.

\section{No extra cost}

What's more, this service will be provided at no extra cost. As in previous years, electronic access to the current volume of each institutionally subscribed title will also be provided at no extra cost.

\section{Electronic Journals: an on-going solution}

Institute of Physics Publishing say that they will continue to invest their time to work within a customer-focused development program, bringing even more value to your print journal. That's an impressive claim, but of course no good scientist takes the answers to difficult questions on trust. You can verify their answers by visiting the company's web site at:

\section{http://www.iop.org}

For further information order a copy of the new IoP leaflet, '5 Easy Steps to Full Text Access'.

\section{Contact Journals marketing quoting ref. AG 0498}

Tel: (800) 3584677 Fax: (215) 6270879

E-mail: collins@ioppubusa.com

or complete and return this coupon:

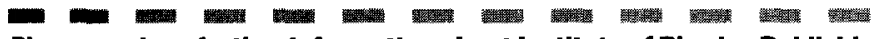
Please send me further information about Institute of Physics Publishing Electronic Journals

Title Name

\begin{tabular}{lll}
\hline Institution & Address \\
\hline Post code & Country & Cel \\
\hline Fax & E-mail & \\
\hline
\end{tabular}

Please return this coupon to Journals marketing, Institute of Physics Publishing, 150 South Independence Mall West, 1035 Public Ledger Building, Philadelphia, PA 19106

\section{Institute of Physics $_{\text {prulshing }}$}

